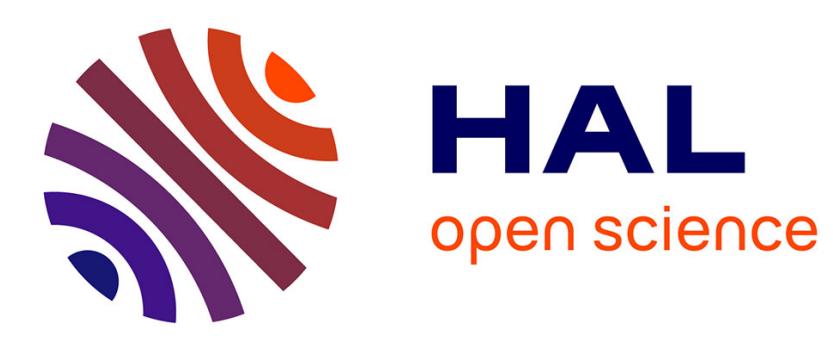

\title{
Production photoélectrochimique d'hydrogène à l'aide de colorants en lumière visible
}

\author{
M. de Backer, M. C. Richoux, F. Leclercq, G. Lepoutre
}

\section{To cite this version:}

M. de Backer, M. C. Richoux, F. Leclercq, G. Lepoutre. Production photoélectrochimique d'hydrogène à l'aide de colorants en lumière visible. Revue de Physique Appliquée, 1980, 15 (3), pp.529-534. 10.1051/rphysap:01980001503052900 . jpa-00244757

\section{HAL Id: jpa-00244757 https://hal.science/jpa-00244757}

Submitted on 1 Jan 1980

HAL is a multi-disciplinary open access archive for the deposit and dissemination of scientific research documents, whether they are published or not. The documents may come from teaching and research institutions in France or abroad, or from public or private research centers.
L'archive ouverte pluridisciplinaire HAL, est destinée au dépôt et à la diffusion de documents scientifiques de niveau recherche, publiés ou non, émanant des établissements d'enseignement et de recherche français ou étrangers, des laboratoires publics ou privés. 


\title{
Production photoélectrochimique d'hydrogène à l'aide de colorants en lumière visible
}

\author{
M. de Backer, M. C. Richoux, F. Leclercq et G. Lepoutre \\ Laboratoire de Chimie Physique, Faculté libre des Sciences et Ecole des Hautes Etudes Industrielles (*), \\ L.A. 253, 13, rue de Toul, 59046 Lille Cedex, France
}

(Reçu le 27 juillet 1979, révisé le 15 novembre 1979, accepté le 19 novembre 1979)

\begin{abstract}
Résumé. - On donne le principe d'une pile photoélectrochimique qui utilise quatre photons visibles par molécule d'eau photolysée. Les réactions photochimiques ont lieu en phase liquide grâce à des colorants photosensibles mis en solution. La régénération des réactifs a lieu aux électrodes. Les résultats obtenus pour la photoréduction de l'eau en hydrogène sont présentés. Les colorants utilisés sont les phthalocyanines de $\mathrm{Zn}, \mathrm{Mg}, \mathrm{Cr}$ et $\mathrm{Ni}$. Les volumes d'hydrogène produits correspondent à plus de 10 utilisations du colorant; de plus celui-ci ne semble pas se dégrader au cours du temps. Les rendements sont de l'ordre de $1 \%$ par rapport au volume d'hydrogène pouvant être produit dans ces conditions, par des photons de longueur d'onde comprise entre 600 et $650 \mathrm{~nm}$.
\end{abstract}

\begin{abstract}
The principle of a photoelectrochemical cell is described. The process uses four photons of visible light to photolyse one water molecule. Photochemical reactions occur in liquid phase using soluble photosensitizers which are regenerated at inert electrodes. The results obtained for the photoreduction of water into hydrogen are presented. The dyes used are $\mathrm{Zn}, \mathrm{Mg}, \mathrm{Cr}$ and $\mathrm{Ni}$ phthalocyanines. The volumes of hydrogen evolved correspond to a turnover of the dye of the order of 10 ; furthermore no degradation is observed after illumination. The yields are of the order of $1 \%$ with respect to the hydrogen volume which can be evolved under these conditions using photons having wavelength between 600 and $650 \mathrm{~nm}$.
\end{abstract}

L'utilisation de l'énergie solaire par les plantes a permis au cours des siècles le stockage de grandes quantités de combustibles que nous utilisons actuellement (charbon, pétrole, gaz naturel). Le processus de la photosynthèse utilise de la lumière de faible énergie (de longueur d'onde aux environs de $600 \mathrm{~nm}$, soit $2 \mathrm{eV}$ ) et la majeure partie de la lumière solaire peut ainsi être utilisée. Ce mécanisme a donc fait ses preuves, et il est.intéressant de savoir si on peut s'en inspirer pour inventer des systèmes artificiels permettant la conversion directe de l'énergie solaire en énergie chimique.

La quantité d'énergie nécessaire pour décomposer une molécule d'eau est $2 \times 1,23 \mathrm{eV}(237 \mathrm{~kJ} /$ mole $)$ dans les conditions d'équilibre thermodynamique. Dans la photosynthèse, il est connu que quatre photons sont utilisés soit 7,6 eV $(732 \mathrm{~kJ} /$ mole $)$ c'est-à-dire trois fois plus qu'il n'est nécessaire. Pour réaliser cette décomposition les plantes disposent de deux systèmes ou photosystèmes (PS), le premier appelé PS I a pour centre actif la chlorophylle et sert à la réduction de l'eau, les espèces réduites étant ensuite utilisées pour

(*) Associé au C.N.R.S. la transformation du gaz carbonique en carbohydrates; le second (PS II) de nature encore inconnue, met en jeu du manganèse et oxyde l'eau pour fournir les électrons au PS I. Un système complexe de membranes et de chaînes d'oxydoréduction permet la régénération des systèmes utilisés.

Les systèmes biologiques nous apprennent qu'il est nécessaire d'avoir un photosensibilisateur $P$ et un catalyseur $\mathrm{C}$ pour l'oxydation comme pour la réduction de l'eau. Nous les distinguerons par l'indice 0 pour l'oxydation et par $r$ pour la réduction.

L'oxydation de l'eau en oxygène peut s'écrire :

$$
2 \mathrm{H}_{2} \mathrm{O}+\mathrm{C}_{0}^{4+} \rightarrow 4 \mathrm{H}^{+}+\mathrm{O}_{2}+\mathrm{C}_{0} \text {. }
$$

Un photosensibilisateur $P_{0}$ est excité par la lumière. L'électron excité laisse un trou qui prendra un des électrons du catalyseur $C_{0}$ et $P_{0}$ sera réduit. La chaîne hypothétique des réactions peut s'écrire :

$$
\begin{gathered}
\mathrm{P}_{0} \stackrel{h v}{\stackrel{h}{\sim}} \mathrm{P}_{0}^{*} \\
4 \mathrm{P}_{0}^{*}+\mathrm{C}_{0} \longrightarrow 4 \mathrm{P}_{0}^{-}+\mathrm{C}_{0}^{4+} .
\end{gathered}
$$

Le catalyseur se trouve réoxydé et peut réagir sur l'eau selon la réaction (1). 
On peut écrire d'une façon similaire la réduction de l'eau en hydrogène

$$
\begin{aligned}
& \mathrm{P}_{\mathrm{r}} \stackrel{h \nu}{\stackrel{h}{\rightarrow}} \mathrm{P}_{\mathrm{r}}^{*} \\
& 2 \mathrm{P}_{\mathrm{r}}^{*}+\mathrm{C}_{\mathrm{r}} \longrightarrow \mathrm{C}_{\mathrm{r}}^{2-}+2 \mathrm{P}_{\mathrm{r}}^{+} \\
& 2 \mathrm{C}_{\mathrm{r}}^{2-}+4 \mathrm{H}^{+} \longrightarrow 2 \mathrm{C}_{\mathrm{r}}+2 \mathrm{H}_{2} .
\end{aligned}
$$

Le photosensibilisateur excité $\mathrm{P}_{\mathrm{r}}^{*}$ est capable de céder un électron au catalyseur qui peut alors réduire le proton en $\mathrm{H}_{2}$.

Les quatre protons qui sont utilisés dans la réaction (6) proviennent de la réaction (1). Pour que le système soit photocatalytique, il faut que les espèces $\mathrm{P}_{\mathrm{r}}^{+}$et $\mathrm{P}_{0}^{-}$puissent redonner $\mathbf{P}_{\mathrm{r}}$ et $\mathbf{P}_{\mathbf{0}}$.

Ceci peut se faire dans une pile photoélectrochimique décrite sur la figure 1 . Les deux systèmes sont séparés par une membrane perméable aux protons.

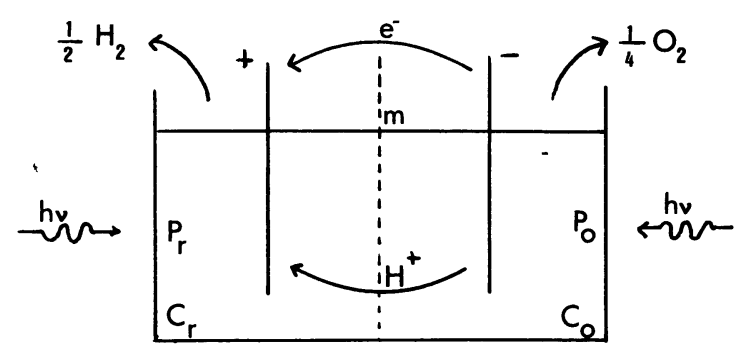

Fig. 1. - Schéma de principe d'une pile photoélectrochimique. $P_{r}$ et $P_{0}$ sont les photosensibilisateurs en solution.

[Diagram of a photoelectrochemical cell. $P_{r}$ and $P_{0}$ are soluble photosensitiźers.]

Deux électrodes plongent dans les solutions; dans le compartiment photoréducteur, l'espèce $P_{r}^{+}$reçoit un électron de l'électrode, alors que du côté photooxydant l'espèce $\mathrm{P}_{0}^{-}$cède un électron à l'électrode. Une différence de potentiel se crée entre les électrodes, les électrons circulent dans un circuit extérieur et permettent ainsi la régénération des colorants [1].

Les réactions aux électrodes sont alors :

$$
\begin{aligned}
\mathrm{P}_{\mathrm{r}}^{+}+\mathrm{e}^{-} & \rightarrow \mathrm{P}_{\mathrm{r}} \\
\mathrm{P}_{0}^{-} & \rightarrow \mathrm{P}_{0}+\mathrm{e}^{-} .
\end{aligned}
$$

Il suffit alors de choisir convenablement les photosensibilisateurs $P_{0}$ et $P_{r}$ en fonction de leurs potentiels redox pour que la régénération soit spontanée.

Dans cet article, nous décrirons les résultats obtenus dans l'étude du système photoréducteur. Dans ce cas, une source extérieure d'électrons est utilisée pour assurer la régénération de $\mathrm{P}_{\mathrm{r}}^{+}$en $\mathrm{P}_{\mathrm{r}}$.

Nous avons reporté sur la figure 2 le schéma de principe du montage.

Les photosensibilisateurs choisis sont les phthalocyanines $(\mathrm{Pc})$ de zinc, chrome et magnésium. Ces colorants ont un motif hydrocarboné analogue à celui de la chlorophylle. Le choix de l'ion métallique central a été dicté par comparaison avec la chlorophylle $(\mathrm{Mg})$

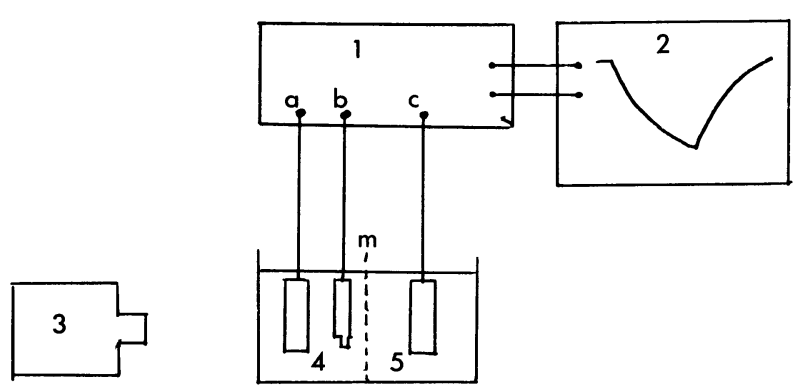

Fig. 2. -1 : Potentiostat, a : électrode de travail, $\mathrm{b}$ : électrode de référence, $\mathrm{c}:$ contre-électrode; 2 : enregistreur de courant; 3 : source lumineuse ; 4 : compartiment éclairé contenant le photosensibilisateur; 5 : compartiment contenant un couple redox; $\mathrm{m}$ : membrane.

[Photoelectrochemical set up. $1:$ potentiostat, a : working electrode, $\mathrm{b}$ : reference electrode, $\mathrm{c}$ : counter electrode; 2 : current recorder ; 3 : light source; 4 : compartment containing the photosensitizers (light) ; 5 : compartment containing a redox couple; m : membrane.]

ou par les résultats que nous avons obtenus en photoélectrochimie (mesure de photopotentiels).

Les phthalocyanines non substituées sont insolubles en milieu aqueux. Pour les utiliser, il faut donc les introduire dans des micelles. On peut également procéder à une sulfonation en vue d'obtenir des produits solubles.

Le catalyseur $\mathrm{C}_{\mathrm{r}}$ utilisé est le méthylviologène : chlorure de $1,1^{\prime}$ diméthyl, $2,2^{\prime}$ bipyridinium $\left(\mathrm{MV}^{2+}\right)$. Le potentiel de la réaction de réduction

$$
\mathrm{MV}^{2+}+\mathrm{e}^{-} \rightarrow \mathrm{MV}^{+}
$$

est de $-0,44 \mathrm{~V} / \mathrm{ENH}$, indépendant du $\mathrm{pH}$. Pour des $\mathrm{pH}$ acides la réaction

$$
2 \mathrm{MV}^{+}+2 \mathrm{H}^{+} \rightarrow \mathrm{H}_{2}+2 \mathrm{MV}^{2+}
$$

est catalysée par le platine divisé ou par l'hydrogénase [2].

Nous donnons figure 3 une échelle des potentiels

$$
\begin{aligned}
& \stackrel{\mathrm{E}^{\circ}}{1,23-} \frac{1}{4} \mathrm{O}_{2}+\mathrm{H}^{+}+\mathrm{e}^{-} \longrightarrow \frac{1}{2} \mathrm{H}_{2} \mathrm{O} \\
& -\mathrm{P}_{\mathrm{r}}^{+}+\mathrm{e}^{-} \rightarrow \mathrm{P}_{\mathrm{r}} \\
& 0,0-\mathrm{H}^{+}+\mathrm{e}^{-} \longrightarrow \frac{1}{2} \mathrm{H}_{2} \\
& -\mathrm{C}_{\mathrm{r}}^{+}+\mathrm{e}^{-} \rightarrow \mathrm{C}_{\mathrm{r}} \\
& -\mathrm{P}_{\mathrm{r}}^{+}+\mathrm{e}^{-} \longrightarrow \mathrm{P}_{\mathrm{r}}^{*} \longleftarrow
\end{aligned}
$$

Fig. 3. - Echelle des potentiels standard de réduction mis en œuvre pour la photoréduction de l'eau (en volts par rapport à l'électrode normale à hydrogène).

[Diagram of the standard reduction potentials used for water photoreduction (volts $v s$ NHE).] 
standards de réduction des divers couples présents en solution. Pour établir cette figure on convient de noter en haut de l'échelle les oxydants forts, en bas les réducteurs forts.

Le potentiel du couple $\mathrm{P}^{*} / \mathrm{P}^{+}$n'est pas directement mesurable mais on peut estimer sa valeur à partir de celle du couple $\mathbf{P} / \mathbf{P}^{+}$à l'état fondamental et des données spectroscopiques [17].' Il faut noter que ces valeurs correspondent à l'état hypothétique molal (activités de chaque constituant prises égales à l'unité) ; le potentiel réel du couple $\mathbf{P}^{*} / \mathbf{P}^{+}$dépendra de la concentration de $\mathrm{P}^{*}$ que l'on a à l'état stationnaire sous éclairement.

Partie expérimentale. - 1. Produits. - Les phthalocyanines non substituées sont des produits commerciaux (Eastman Kodak); elles sont purifiées par chauffage sous vide à $200^{\circ} \mathrm{C}$. Les phthalocyanines sulfonées doivent être synthétisées directement pour éviter les problèmes de démétallation qui se produisent lors des sulfonations directes à l'aide d'oléum d'acide sulfurique. La synthèse est réalisée en faisant réagir à $180^{\circ} \mathrm{C}$ de l'acide sulfophthalique avec de l'urée en présence d'un sel du métal que l'on veut y introduire. Nous nous sommes inspirés de la méthode de Rollmann et Iwamoto [3] pour la synthèse et de celle de Bernauer et Fallab [4] pour la purification. Le produit final obtenu n'est pas d'une pureté suffisante pour qu'une micro-analyse donne de bons résultats. Nous estimons les concentrations d'après l'absorbance des solutions. Les solutions utilisées ont une absorbance comprise entre 1 et 2 , ce qui correspond à une concentration de l'ordre de $10^{-5} \mathrm{M}[3,4]$. Les impuretés ne sont pas colorées et ne sont pas actives électrochimiquement.

Nous avons aussi utilisé des dispersions de phthalocyanines dans des micelles anioniques. Le tensio actif utilisé était le dodécyl sulfate de sodium (SDS); la concentration micellaire critique est $8,3 \times 10^{-3} \mathrm{M}$ et un nombre moyen de 60 molécules de tensio-actif s'agrègent pour former les micelles [5].

Afin de pouvoir effectuer des mesures électrochimiques, un électrolyte support $\mathrm{NaCl}$ ou $\mathrm{KCl}$ est ajouté à la solution (les micelles de SDS précipitent en présence de $\mathrm{K}^{+}$). Le $\mathrm{pH}$ de la solution est ajusté à l'aide de solutions tampon phosphate-citrate ou de BrittonRobinson [6].

Le méthylviologène (Aldrich) est utilisé sans autre purification. Des analyses polarographiques ont montré qu'il n'existait pas d'impuretés électroactives dans le domaine électrochimique étudié. Le catalyseur pour la réaction de production d'hydrogène est soit $\mathrm{du} \mathrm{Na}_{2} \mathrm{PtCl}_{6}$ en solution, du platine colloïdal [7] ou $\mathrm{du} \mathrm{PtO}_{2}$.

2. Montage PHotó́lectrochimiQue. - Nous utilisons un montage potentiostatique à trois électrodes. L'électrode contrôlée est un disque de platine platiné de $25 \mathrm{~mm}$ de diamètre, l'électrode de référence est une électrode au calomel saturé, la contre-électrode est constituée par une grille de platine. Le compartiment de la contre-électrode est séparé du compartiment de travail par un pont salin rempli d'électrolyte support.

La cellule est représentée sur la figure 4. Le compartiment de mesure est étanche ; il est muni d'un septum permettant l'échantillonnage des gaz. Le volume de la solution contenue dans la cellule est d'environ $150 \mathrm{ml}$, et celui du gaz est de $75 \mathrm{ml}$. La cellule est thermostatée par une circulation d'eau qui sert également de filtre infrarouge.

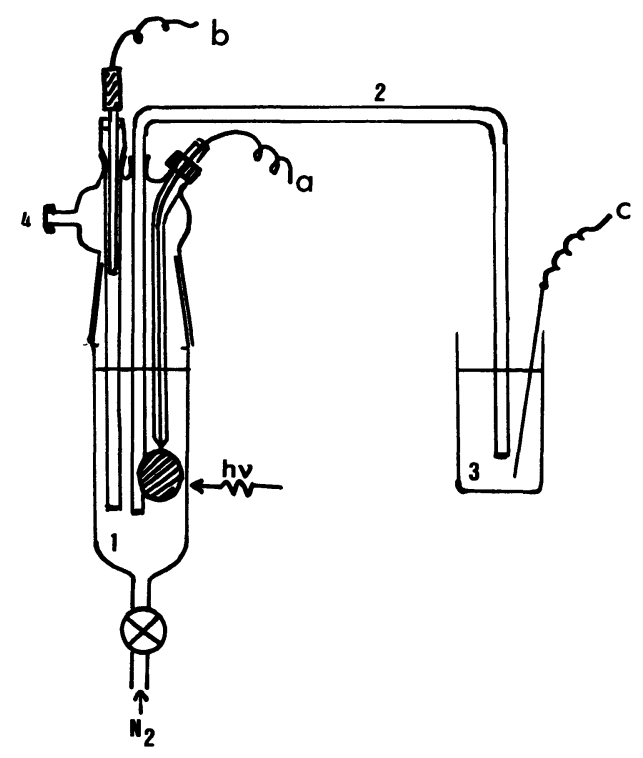

Fig. 4. - Schéma de la cellule utilisée : a, b, c, comme figure 2 ; 1 : compartiment contenant le photosensibilisateur; 2 : pont salin ; 3 : solution d'un couple redox.

[Drawing of the cell used : $a, b, c:$ as on figure $2 ; 1:$ compartment containing the photosensitizer; 2 : salt bridge $; 3:$ redox couple in solution.]

La source lumineuse est constituée d'un projecteur de diapositives équipé d'une lampe quartz-halogène de $250 \mathrm{~W}$ sous $24 \mathrm{~V}$. La lumière est focalisée sur l'électrode à l'aide d'une lentille. L'énergie lumineuse incidente est alors de $250 \mathrm{~mW} / \mathrm{cm}^{2}$, cette valeur est mesurée à l'aide d'un pyranomètre et tient compte de la partie infrarouge du rayonnement. Cette valeur ne tient pas compte des réflexions sur la double paroi cylindrique de la cellule. Nous avons effectué nos expériences en lumière blanche. (Nous avons néanmoins vérifié que la lumière rouge était encore efficace.) A l'exception de la lampe, toute la partie optique est réalisée en verre ou en pyrex, ce qui nous permet d'éliminer la partie ultraviolette de la lumière.

3. DoSAGE DE L'HYDRogène. - L'hydrogène formé est prélevé dans le volume gazeux de la cellule à l'aide d'une seringue à gaz de $100 \mu \mathrm{l}$. Le gaz (mélange $\mathrm{H}_{2}-\mathrm{N}_{2}$ ) est analysé par chromatographie en phase gazeuse sur une colonne garnie de tamis moléculaire $5 \AA$; le gaz vecteur est l'argon. L'étalonnage de l'appareil 
est réalisé à l'aide de mélanges $\mathrm{H}_{2}-\mathrm{N}_{2}$ dont la composition est proche de celle des mélanges inconnus. La surface du pic chromatographique de l'azote sert de référence pour normer les chromatogrammes.

4. DESCRIPTION D'UNE EXPÉRIENCE TYPE. - On introduit dans la cellule la solution à étudier (phthalocyanine, méthylviologène, catalyseur, électrolyte support) portée au $\mathrm{pH}$ désiré à l'aide de solutions tampon. On chasse l'oxygène par un balayage d'azote $\mathrm{R}$ pendant au moins $30 \mathrm{~min}$. Après arrêt du courant d'azote, le potentiel choisi est appliqué à l'électrode de travail ; on mesure le courant et l'on attend qu'il se stabilise. Après plusieurs heures une prise de gaz est effectuée pour s'assurer qu'il n'y a pas eu production d'hydrogène. La solution est alors éclairée, un photocourant est observé et des analyses de gaz sont faites au cours du temps. Il faut noter que le photocourant met plusieurs minutes à s'établir, ce qui est contraire aux résultats observés lorsque l'on éclaire des électrodes photosensibles.

Résultats. - Dans cet article, nous donnerons les résultats de photoproduction d'hydrogène. Les photocourants en fonction du temps et du potentiel imposé ont aussi été mesurés et les résultats obtenus sont similaires à ceux que nous avons déjà publiés [8].

Les photocourants obtenus sont de l'ordre de $100 \mu \mathrm{A}$ ce qui correspond à une production d'hydrogène de $0,04 \mathrm{ml} \mathrm{H}_{2} \mathrm{~h}^{-1}$ si le passage des électrons sert exclusivement à la production d'hydrogène.

Nous avons d'abord imposé à l'électrode de travail d'être à un potentiel peu éloigné de celui de l'électrolyse, c'est-à-dire à la valeur la plus négative que nous puissions fixer. Le potentiel thermodynamique d'électrolyse est $E=-0,059 \mathrm{pH}$. A ce potentiel, compte tenu des surtensions aux électrodes, la quantité d'hydrogène produite à l'obscurité doit être faible.

Pour cette valeur du potentiel, la régénération de la forme oxydée du colorant est plus efficace (réaction 7 déplacée vers la droite). De même la réoxydation à l'électrode de travail du méthylviologène radicalaire (réaction parasite) est alors peu importante. Si on place l'électrode de travail au potentiel thermodynamique d'équilibre entre le proton et l'hydrogène gaz, on parle non plus de photolyse mais plutôt d'électrolyse'photo-assistée (voir Fig. 3).

Notre plan de travail était donc le suivant : on étudie un système donné à l'obscurité puis à la lumière en imposant à l'électrode de travail d'être proche du potentiel thermodynamique. Si la production d'hydrogène est plus forte à la lumière qu'à l'obscurité, on étudie le comportement du système en imposant à l'électrode d'être à un potentiel de plus en plus oxydant par rapport à la valeur du potentiel thermodynamique. Nous avons regroupé dans le tableau I les résultats obtenus pour différents systèmes utilisant des solutions micellaires de MgPc.

Les expériences 1 et 2 ont été réalisées sans méthylviologène. L'expérience 1 a été réalisée au potentiel thermodynamique de l'hydrogène tandis que pour l'expérience 2 le potentiel était de $60 \mathrm{mV}$ plus oxydant que le potentiel d'électrolyse.

Les expériences 3 et 4 faisant intervenir un système complet : colorant, catalyseur et couple transfert ont été réalisés respectivement à 20 et $40 \mathrm{mV}$ du potentiel d'électrolyse.

En raison de problèmes expérimentaux, la quantité d'hydrogène n'a pu être mesurée qu'en fin d'expérience. On mesure d'abord l'hydrogène obtenu à la fin de la période d'éclairement puis après purge de la cellule on mesure la production d'hydrogène à l'obscurité au même potentiel. On note que le rapport entre $\mathrm{H}_{2}$ mesuré à la lumière et $\mathrm{H}_{2}$ mesuré à l'obscurité est beaucoup plus important lorsque le potentiel imposé est plus éloigné du potentiel thermodynamique.

La figure 5 donne la production d' $\mathrm{H}_{2}$ en fonction du temps pour la phthalocyanine tétrasulfonée de chrome. Les expériences ont duré environ $50 \mathrm{~h}$; elles ont été interrompues au bout de ce laps de temps en raison des entrées d'air qui se produisent au niveau du septum après de nombreux prélèvements de gaz.

On remarque que, dans les deux cas présentés, la production d'hydrogène se poursuit à l'obscurité.

Ce phénomène s'observe d'autant mieux qu'il y a eu auparavant une période d'illumination assez longue avec production importante d' $\mathrm{H}_{2}$.

La production d'hydrogène par électrolyse ne pouvant se faire au potentiel imposé, on est amené à supposer une accumulation d'espèces réduites durant

Tableau I.

\begin{tabular}{|c|c|c|c|c|c|c|c|c|c|}
\hline \multirow[b]{2}{*}{ No } & & \multirow[b]{2}{*}{$\mathrm{pH}$} & \multirow[b]{2}{*}{$E_{\mathrm{H}^{+} / \mathrm{H}_{2}}\left({ }^{1}\right)$} & \multirow[b]{2}{*}{$E_{\text {imposé }}$} & \multicolumn{2}{|c|}{ Obscurité } & \multicolumn{2}{|c|}{ Lumière } & \multirow{3}{*}{ Rapport } \\
\hline & & & & & $\mathrm{ml} \mathrm{H}_{2} / \mathrm{h}$ & Durée & $\mathrm{ml} \mathrm{H}_{2} / \mathrm{h}$ & Durée & \\
\hline - & & - & - & - & - & - & - & - & \\
\hline 1 & $\operatorname{MgPc} \operatorname{SDS}(4,66 \mathrm{~g} / 1)$ & 4,2 & -500 & -500 & 0,031 & $4 \mathrm{~h}$ & 0,234 & $15 \mathrm{~h}$ & 7,8 \\
\hline 2 & $\operatorname{MgPc} \operatorname{SDS}(7 \mathrm{~g} / 1)$ & 5,8 & -600 & -540 & 0,0006 & $15 \mathrm{~h}$ & 0,0125 & $7 \mathrm{~h}$ & 20,8 \\
\hline 3 & $\begin{array}{l}\text { MgPc SDS }(15 \mathrm{~g} / 1) \\
+ \text { colloïdal } P t \\
+\mathrm{MV}^{++} 2 \times 10^{-4} \mathrm{M}\end{array}$ & 4,0 & -490 & -470 & 0,39 & $20 \mathrm{~h} 15 \mathrm{~min}$ & 0,57 & $18 \mathrm{~h} 10 \mathrm{~min}$ & 1,6 \\
\hline 4 & $\begin{array}{l}\text { MgPc SDS }(15 \mathrm{~g} / 1) \\
+ \text { colloïdal } P t \\
+\mathrm{MV}^{++} 2 \times 10^{-4} \mathrm{M}\end{array}$ & 4,0 & -490 & -450 & 0,001 & $2 \mathrm{~h} 15 \mathrm{~min}$ & 0,021 & $3 \mathrm{~h}$ & 21 \\
\hline
\end{tabular}

${ }^{1}$ ) Les potentiels sont en $\mathrm{mV}$ par rapport à l'électrode au calomel saturé. 


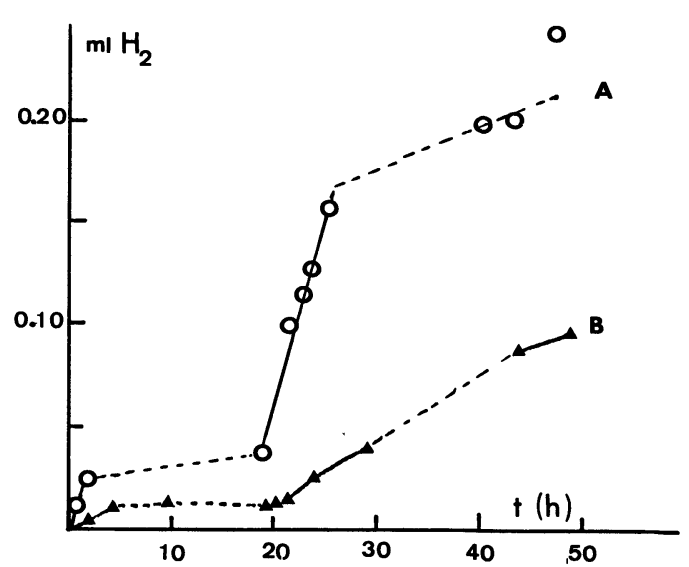

Fig. 5. - Dégagement d'hydrogène en fonction du temps. Photosensibilisateur : phthalocyanine de chrome sulfonée $(40 \mathrm{mg} / 1)$; catalyseur méthylviologène $\left(10^{-3} \mathrm{M}\right) . \mathrm{A}: E_{\mathrm{imp}}=-0,45 \mathrm{~V} / \mathrm{ECS}$; $\mathrm{pH}=4,2 ;$ Pt colloïdal. B : $E_{\text {imp }}=-0,53 \mathrm{~V} / \mathrm{ECS} ; \mathrm{pH}=6$, $\mathrm{Na}_{2} \mathrm{PtCl}_{6}$. Les pointillés indiquent les périodes à l'obscurité.

[Hydrogen evolution as a function of time. Sulfonated $\mathrm{Cr}$ phthalocyanin $\left(40 \mathrm{mg}\right.$ 's); methylviologen $\left(10^{-3} \mathrm{M}\right)$.

$$
\mathrm{A}: E_{\mathrm{imp}}=-0.45 \mathrm{~V} / \mathrm{SCE} ; \mathrm{pH}=4.2 ; \quad \mathrm{Pt} \text { colloidal. }
$$

B : $E_{\text {imp }}=-0.53 \mathrm{~V} / \mathrm{SCE} ; \mathrm{pH}=6 ; \mathrm{Na}_{2} \mathrm{PtCl}_{6}$. Dotted lines indicate dark periods.]

la période d'éclairement, qui continuerait le processus de dégagement pendant un certain temps, et une longue période de mise en équilibre gaz-solution. Il faut également tenir compte de ce phénomène lors de l'exploitation des expériences résumées dans le tableau I.

La figure 6 met en évidence l'influence de l'addition de méthylviologène sur la vitesse de dégagement de l'hydrogène à partir d'une solution de PcZn.

D'autres essais similaires ont été réalisés en utilisant $\mathrm{NiPc}$ et ZnPc. Ces phthalocyanines permettent aussi d'obtenir un dégagement d'hydrogène mais pour l'instant nous ne sommes pas parvenus à travailler à plus de $80 \mathrm{mV}$ de l'électrolyse.

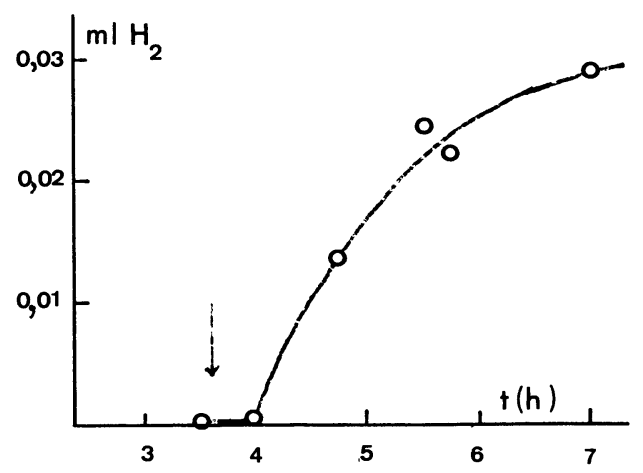

Fig. 6. - Influence de l'addition de méthylviologène sur la vitesse de dégagement de l'hydrogène. Phthalocyanine de zinc sulfonée $(40 \mathrm{mg} / 1) E_{\mathrm{imp}}=-0,55 \mathrm{~V} / \mathrm{ECS} ; \mathrm{pH}: 6,05 ; \mathrm{PtO}_{2}$.

[Influence of methylviologen on the rate of hydrogen evolution Sulfonated zinc phthalocyanin $(40 \mathrm{mg} / 1) E_{\text {imp }}=-0.55 \mathrm{~V} / \mathrm{SCE}$; $\mathrm{pH}=6,05 ; \mathrm{PtO}_{2}$.]
Nous avons aussi obtenu des résultats positifs en remplaçant les catalyseurs à base de platine par une hydrogénase [9]. Nous avons également réalisé quelques essais sans méthylviologène afin que l'électrochimie des solutions soit plus facile à étudier. Dans ce dernier cas, une production d'hydrogène peut parfois être obtenue mais les résultats ne sont pas reproductibles pour l'instant.

Discussion. - Nous avons montré qu'il était possible de réduire l'eau en hydrogène en utilisant les phthalocyanines de $\mathrm{Zn}, \mathrm{Cr}, \mathrm{Mg}$, comme photosensibilisateurs, de la lumière visible, et une régénération électrochimique des colorants utilisés.

Les volumes d'hydrogène dégagés correspondent à plusieurs utilisations du colorant. En effet, la quantité de colorant contenue dans la cellule est de l'ordre de $2 \times 10^{-6}$ moles. Si l'hydrogène provenait de la destruction de ce colorant, le volume maximum serait de $2,24 \times 10^{-2} \mathrm{ml}$. Les volumes obtenus montrent que dans la plupart des cas, la phthalocyanine a servi une dizaine de fois. L'étude des spectres visibles de la solution avant et après les manipulations montre que le colorant ne se consomme pas.

Nous n'avons pas encore réalisé d'essais en vue de connaître le nombre limite de cycles possibles, ceci est dû à la faible efficacité du système qui nous force à réaliser des expériences de longue durée et aussi à l'empoisonnement du système par l'air introduit lors des nombreuses piqûres à travers le septum.

Quand il n'y a pas en solution d'espèces réductibles autres que l'eau, le photocourant mesuré correspond bien à la quantité d'hydrogène dégagée. Ainsi dans le cas de la courbe $A$ (Fig. 4), la quantité d'hydrogène correspondant au photocourant est de $0,37 \mathrm{ml}$ alors que la quantité mesurée est de $0,25 \mathrm{ml}$. Dans le cas de la courbe $\mathrm{B}$, une grande quantité de $\mathrm{Pt}^{4+}$ est présente dans la solution, et le courant observé ne correspond plus à la régénération du colorant mais en majeure partie à la réduction de $\mathrm{Pt}^{4+}$ à l'électrode.

Pour évaluer le rendement de la réaction par rapport à l'énergie lumineuse reçue, nous calculons le volume maximum d'hydrogène que l'on peut produire. La cellule reçoit une énergie lumineuse de $0,25 \mathrm{~W} . \mathrm{cm}^{-2}$, répartie sur tout le spectre y compris l'infrarouge. La lumière active dans notre système a une longueur d'onde comprise entre 600 et $650 \mathrm{~nm}$. En assimilant la lampe à un corps noir porté à $3500 \mathrm{~K}$, on calcule que $4 \%$ de l'énergie totale émise se trouve dans la gamme utile, soit $10^{-2} \mathrm{~W} \cdot \mathrm{cm}^{-2}\left(10^{-7} \mathrm{eV} \cdot \mathrm{cm}^{-2} \mathrm{~s}^{-1}\right)$.

Dans le processus de photoréduction de l'eau, deux photons d'énergie moyenne $2 \mathrm{eV}$ sont utilisés pour former une molécule d'hydrogène. L'utilisation totale de la lumière incidente conduirait à une production d'hydrogène de $2 \mathrm{ml} . \mathrm{h}^{-1} \mathrm{~cm}^{-2}$. La vitesse de dégagement maximum que nous avons observée

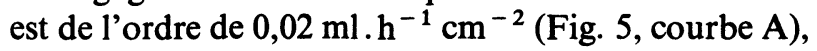
ce qui correspond à un rendement de $1 \%$ par rapport à la lumière incidente de longueur d'onde comprise 
entre 600 et $650 \mathrm{~nm}$. Dans cette estimation, nous avons négligé les pertes de lumière causées par réflexion et transmission; elles contribuent à diminuer le rendement.

D'autres auteurs [10-16] ont réalisé la photodécomposition de l'eau en phase homogène. Le principe de la réduction est le même que celui que nous avons décrit ; la principale différence réside dans la méthode de régénération des colorants photooxydés (réaction 7). Dans notre procédé, nous fournissons les électrons nécessaires, d'une manière contrôlée par l'intermédiaire d'électrodes, alors que les procédés en phase homogène nécessitent un donneur d'électron supplémentaire (EDTA ou triéthanolamine) qui est consommé de manière irréversible.

En principe, le potentiel appliqué à l'électrode plongée dans la solution devrait être tel que la réaction 7 se fasse à l'électrode. Un potentiel plus négatif que le $E_{0}$ de cette réaction assurerait que le photosensibilisateur se trouve sous forme réduite. Ceci est nécessaire pour que des réactions parasites telles que

$$
\mathrm{P}_{\mathrm{r}}^{+}+\mathrm{C}_{\mathrm{r}}^{-} \rightarrow \mathrm{P}_{\mathrm{r}}+\mathrm{C}_{\mathrm{r}}
$$

ne deviennent trop importantes. En outre, il est souhaitable que ce potentiel soit aussi éloigné que possible du potentiel de réduction du proton si l'on veut que des économies d'énergie soient réalisées. Il faut aussi considérer que dans un système complet c'est la photo-oxydation de l'eau qui fournira les électrons au potentiel nécessaire pour que le système puisse fonctionner seul. Le choix de ce potentiel doit se faire en sélectionnant le colorant.

Jusqu'à présent, nous avons dû travailler à des potentiels peu éloignés de celui de l'électrolyse.

Nous nous attacherons donc dans la suite de l'étude à rechercher d'autres colorants qui permettraient de travailler à des potentiels moins réducteurs.

Il sera nécessaire d'étudier les potentiels redox de ces colorants dans l'eau ainsi que l'influence des substituants du cycle sur ces potentiels. D'autre part, il faudra déterminer les cinétiques des réactions aux électrodes afin de connaître l'interaction entre l'électrode et les couples tels que $\mathrm{H}^{+} / \mathrm{H}_{2}$ ou $\mathrm{MV}^{+} / \mathrm{MV}^{++}$ présents dans la solution.

Ce travail a bénéficié du support de la CEE contrat 533-78 ESF. L'une d'entre nous ( $F$. Leclercq) a bénéficié d'une allocation de recherche de la DGRST.

\section{Bibliographie}

[1] Lepoutre, G. et De Backer, M. G., Colloq. Int. Electricité Solaire, Toulouse 453 (mars 1976)

DE BACKer, M. G., Richoux, M. C., LeClercQ, F. and Lepoutre, G., Int. Conf. Solar Energy, Cambridge, 51 (août 1978).

[2] Yu, L. and Wolliw, M. J., J. Bacteriol. 98 (1969) 1, 51.

[3] Rollmann, L. D. and Iwamoto, R. T., J. Am. Chem. Soc. 90 (1968) 6, 1455.

[4] Bernauer, K. and Fallab, S., Helv. Chim. Acta 44 (1961) 1287.

[5] Heinglein, A. and Gratzel, M., in Solar Power and Fuels, J. R. Bolton, Ed. (Acad. Press, Inc., New York, N.Y.) 1977, chap. 3.

[6] Britton, H. T. S. and Robinson, R. A., J. Chem. Soc. (1931) 1454

[7] Rampino, L. D. and Nord, F. F., J. Am. Chem. Soc. 63 (1941) 2745.

[8] Cappelle, P., De Backer, M. G., De Whitte, O., Feuillade, G. et Lepoutre, G., C.R. Hebd. Séan. Acad. Sci. 284 (1977) 16, 597.

[9] Don du Professeur D. O. Hall, University of London King's College.
[10] Moradpour, A., Amouyal, E., Keller, P. and Kagaw, H., Nouv. J. Chim. 2 (1978) 547.

[11] Lehn, J. M. and Sauvage, J. P., Nouv. J. Chim. 1 (1977) 449. Kirch, M., Lehn, J. M. and Sauvage, J. P., Helv. Chim. Acta 62 (1979) 4, 1345.

[12] Koriakin, B. V., Dzhabiev, T. S. and Shilov, A. E., Dokl. Akad. Nauk S.S.R.R. 233 (1977) 620.

[13] Krasna, A. I., in Biological Solar Energy Conversion. San Petro, A. and Mitsuri, A. (Eds. Academic Press, New York) 1977.

Krasna, A. I., Photochem. Photobiol. 29 (1979) 267.

[14] ManN, R. R., Lewis, N. S., Miskowski, V. M., ERwin, D. K., Hammond, G. S. and Gray, H. B., J. Am. Chem. Soc. 99 (1977) 5525.

[15] Kalyanasundaram, K., Kiwi, J. and Gratzel, M., Helv. Chim. Acta 61 (1978) 2720.

[16] Okura, I. and Kim-Thuan, N., J. Mol. Catalysis 5 (1979) 31. Brown, G. M., Brunschwig, B. S., Creutz, C., EndicotT, J. F. and Sutin, N., J. Am. Chem. Soc. 101 (1979) 1298

[17] Seely, G. R. in The Chlorophylls, G. R. Seely ed. (Acad. Press, New York) 1966. 